\title{
Judicialização da saúde: conhecimentos de estudantes de enfermagem, medicina e direito
}

\author{
Judicialization of health: knowledge of nursing, medicine and law students \\ Judicialización de la salud: conocimientos de estudiantes de enfermería, medicina y derecho
}

Recebido: 09/02/2021 | Revisado: 16/02/2021 | Aceito: 17/02/2021 | Publicado: 27/02/2021

Gabriela do Rosário Paloski

ORCID: https://orcid.org/0000-0003-3391-2076

Universidade Federal do Rio Grande, Brasil

E-mail: gabipaloski@outlook.com

Jamila Geri Tomaschewski Barlem

ORCID: https://orcid.org/0000-0001-9125-9103

Universidade Federal do Rio Grande, Brasil

E-mail: jamila_tomaschewski@hotmail.com

Janaína Sena Castanheira

ORCID: https://orcid.org/0000-0002-8300-698X

Universidade Federal do Rio Grande, Brasil

E-mail: janainasena@ furg.br

Aline Neutzling Brum

ORCID: https://orcid.org/0000-0002-9686-9602

Universidade Federal do Rio Grande, Brasil E-mail: neutzling@live.de

Cristiane Lima de Moraes

ORCID: https://orcid.org/0000-0002-9479-2867

Universidade Federal do Rio Grande, Brasil

E-mail: krismoraes31@hotmail.com

Glaucia Dal Omo Nicola

ORCID: https://orcid.org/0000-0003-1337-6739

Universidade Federal do Rio Grande, Brasil

E-mail: glaucianicola@hotmail.com

\begin{abstract}
Resumo
Este estudo teve por objetivo analisar o conhecimento de estudantes de Enfermagem, Medicina e Direito acerca da judicialização da saúde. Trata-se de um estudo quantitativo, exploratório e descritivo, realizado com 117 estudantes dos cursos de enfermagem, medicina e direito, por meio da aplicação de um questionário elaborado pelos autores do trabalho. A coleta de dados ocorreu no mês de março a maio de 2019. A análise ocorreu por meio do software estatístico Statistical Package for Social Sciences versão 22.0. Com base nos resultados, em relação à formação acadêmica, os estudantes apontam que há necessidade de maior ênfase no desenvolvimento de pensamento ético, crítico e reflexivo para a judicialização da saúde. Dessa forma, pode-se concluir que há necessidade de discussão e ampliação dos espaços de formação acadêmica e profissional dos cursos da saúde e direito acerca da judicialização da saúde.
\end{abstract}

Palavras-chave: Judicialização da saúde; Estudantes de enfermagem; Estudantes de medicina; Estudantes do direito; Saúde.

\begin{abstract}
This study aimed to analyze the knowledge of Nursing, Medicine and Law students about the judicialization of health. This is a quantitative, exploratory and descriptive study, carried out with 117 students from the nursing, medicine and law courses, through the application of a questionnaire prepared by the authors of the work. Data collection took place from March to May 2019. The analysis took place using the statistical software Statistical Package for Social Sciences version 22.0. Based on the results, in relation to academic training, students point out that there is a need for greater emphasis on the development of ethical, critical and reflective thinking for the judicialization of health. Thus, it can be concluded that there is a need for discussion and expansion of spaces for academic and professional training in health and law courses regarding the judicialization of health.
\end{abstract}

Keywords: Health judicialization; Nursing students; Medical students; Law students; Health.

\section{Resumen}

Este estudio tuvo como objetivo analizar los conocimientos de los estudiantes de Enfermería, Medicina y Derecho sobre la judicialización de la salud. Se trata de un estudio cuantitativo, exploratorio y descriptivo, realizado con 117 
estudiantes de los cursos de enfermería, medicina y derecho, mediante la aplicación de un cuestionario elaborado por los autores del trabajo. La recolección de datos se realizó de marzo a mayo de 2019. El análisis se realizó mediante el software estadístico Statistical Package for Social Sciences versión 22.0. Con base en los resultados, en relación a la formación académica, los estudiantes señalan que existe la necesidad de un mayor énfasis en el desarrollo de un pensamiento ético, crítico y reflexivo para la judicialización de la salud. Así, se puede concluir que existe la necesidad de discusión y ampliación de espacios de formación académica y profesional en los cursos de salud y derecho en materia de judicialización de la salud.

Palabras clave: Judicialización de la salud; Estudiantes de enfermería; Estudiantes de medicina; Estudiantes de derecho; Salud.

\section{Introdução}

O conceito de saúde começou a ser difundido em 1946, quando a Organização Mundial da Saúde (OMS) conceituou saúde como sendo um estado completo de bem-estar físico, mental e social e não apenas ausência de doença. A Declaração Universal dos Direitos Humanos de 1948 e o Protocolo sobre Direitos Econômicos, Sociais e Culturais de 1988 consideram a saúde uma condição essencial à dignidade humana, que está totalmente relacionada com as condições socioambientais e econômicas do indivíduo, sendo dever do Estado dar condições progressivas para garantia desse direito (Travassos et al., 2013).

No Brasil, somente após a Constituição Federal da República de 1988, que a saúde passou a ser garantida como um direito da população, assegurando desta maneira como um direito igualitário e universal (Calliari \& Teixeira, 2017). O direito à saúde é reconhecido como direito humano fundamental à preservação da vida, por isso, acredita-se que o direito à saúde é uma obrigação moral e legal de ser cumprido (Lopes et al., 2019).

A judicialização tem sido utilizada como uma maneira rápida de garantir a efetivação dos direitos constitucionais, e pode ser definida como um conjunto de reivindicações por parte dos cidadãos ao Poder Judiciário, a fim de garantir direitos e bens. Essas reivindicações podem estar relacionadas com a obtenção de medicamentos de alto custo, acesso a um determinado serviço de saúde, entre outras demandas garantidas pelo princípio do direito à saúde (Andia \& Lamprea, 2019).

Nota-se que nos últimos 30 anos, houve um aumento no número de processos judiciais relacionados ao direito à saúde, sendo que entre os anos de 2016 e 2017 esse aumento foi de 50\% (Paixão, 2019). Assim, uma forma de reduzir o número de ações judiciais ligados à saúde é abordar a temática sobre judicialização em saúde desde a formação dos estudantes de enfermagem, medicina e do direito (Oates et al, 2018; Souza et al., 2018; Wegner et al., 2016), visto que ao discutir questões de judicialização na formação profissional pode contribuir para uma formação em direito mais crítico-reflexiva, com melhor discernimento sobre os processos jurídicos, e uma formação em enfermagem e medicina mais ética e com uma visão mais holística, diminuindo a violação de direitos.

Diante isso, torna-se importante analisar o que os estudantes conhecem acerca da judicialização da saúde, permitindo maior compreensão sobre o papel destes na garantia da não violação dos direitos ao acesso aos serviços e à saúde dos usuários do Sistema Único de Saúde (SUS). Logo, considerando a escassez de pesquisas que abordem a temática judicialização em saúde na formação de estudantes de enfermagem, medicina e direito, propõem-se a realização deste estudo. Esse estudo apresenta como questão problema: qual o conhecimento dos estudantes de Enfermagem, Medicina e Direito acerca da judicialização da saúde?

Portanto, este estudo tem por objetivo analisar o conhecimento de estudantes de Enfermagem, Medicina e Direito acerca da judicialização da saúde. 


\section{Metodologia}

Trata-se de um estudo exploratório-descritivo com delineamento transversal, realizado com 117 estudantes do quinto ano dos cursos da graduação de Enfermagem, Medicina e Direito de uma Universidade Federal do Sul do Brasil.

O delineamento desse estudo caracterizou-se como transversal, tendo em vista que os dados foram obtidos em um único período de tempo. A pesquisa quantitativa estima a medição de variáveis anteriormente estabelecidas, buscando esclarecer sua influência sobre outras variáveis, concentrando-se em dados numéricos e exatos (Appolinário, 2012). A pesquisa do tipo exploratório-descritivo tem por objetivo a demarcação de características de determinado grupo ou população, direcionando investigações que pretendem analisar determinados fenômenos, caracterizando variáveis envolvidas, esclarecendo relações entre essas e confirmando hipóteses (Gray, 2012).

A coleta de dados foi realizada nos meses de março a maio de 2019, por meio da aplicação de uma escala sobre judicialização da saúde para estudantes de Enfermagem, Medicina e Direito, criada pelos autores deste trabalho.

A seleção do tamanho amostral foi definida pela utilização de uma fórmula específica que estima o mínimo de participantes, uma vez que procedimentos estatísticos diferentes apresentam necessidades específicas de número de participantes (Hill \& Hill, 2012). O número de estudantes matriculados no quinto ano dos cursos de enfermagem, medicina e direito, era de, respectivamente, 32, 59 e 40, sendo considerado o mínimo amostral, após aplicação da fórmula, de respectivamente, 30, 51 e 36. Como critérios de inclusão dos participantes foram considerados estudantes regularmente matriculados no curso de Graduação em Enfermagem, Medicina ou Direito Diurno e que estivessem matriculados em, pelo menos, uma disciplina do quinto ano do seu respectivo curso. Os critérios de exclusão foram à ausência do estudante no local e no momento de coleta de dados por falta, atestados ou licenças. Todos os participantes assinaram o Termo de Consentimento Livre e Esclarecido.

Como instrumento de coleta foi utilizado um questionário, composto por 13 questões em formato Likert de cinco pontos, elaborado pelos autores do trabalho, sendo: "1- discordo totalmente", "2- discordo mais que concordo", “3- não concordo nem discordo", "4- concordo mais que discordo" e "5- concordo totalmente", que os participantes do estudo responderam de acordo com seu grau de concordância (Hill \& Hill, 2012). O instrumento elaborado tinha como objetivo analisar o conhecimento de estudantes de Enfermagem, Medicina e Direito acerca da Judicialização da saúde, abordando afirmativas referentes ao direito à saúde; ao fato das ações judiciais possibilitarem a efetivação do direito à saúde; a garantia de medicamentos de alto custo por meio de processos judiciais; a obtenção de leitos de internação; ao acesso às ações e serviços de saúde; ao acesso à informação; o preparo dos estudantes de enfermagem e medicina obtido a partir da formação acadêmica para lidar com a judicialização em saúde; e, por fim, preparo dos estudantes de direito obtido a partir da formação acadêmica para lidar com a judicialização em saúde.

A aplicação da escala aconteceu mediante agendamento prévio com os professores das disciplinas, com o intuito de realizar a coleta na sala de aula com os estudantes presentes. Estes foram informados que a participação ou não na pesquisa era livre.

Para análise dos dados foi utilizado o software estatístico Statistical Package for Social Sciences (SPSS-IBM) - versão 22.0, por meio da estatística descritiva e da análise de variância. Os dados descritivos foram apresentados por meio de frequências absolutas (n) e relativas (\%), medidas de tendência central (máximo e mínimo) e medidas de dispersão (média e desvio padrão). Para comparação das médias das variáveis contínuas entre os distintos cursos, utilizou-se a análise de variância (ANOVA), o nível de significância adotado foi de $\alpha<0,05$. Este processo facilitou a organização dos dados, o qual será apresentado em tabela para permitir uma melhor visualização dos resultados e sua interpretação.

O estudo foi aprovado pelo Comitê de Ética e Pesquisa (CEP) recebendo aprovação. CAAE: 03580918.3.0000.5324. 


\section{Resultados e Discussão}

Entre os estudantes que participaram da pesquisa, 30 eram da enfermagem, 51 da medicina e 36 do direito, totalizando 117 participantes. Com relação a idade dos participantes observou-se que a média entre os três cursos foi de 25,8 anos, sendo 28,7 entre os estudantes de enfermagem, 24,7 entre os estudantes de medicina e 25,3 anos entre os estudantes do curso de direito.

As médias que serão apresentadas se referem ao nível de concordância dos estudantes acerca do conhecimento sobre a judicialização da saúde. Dessa forma, análise descritiva, como pode ser visualizado na Tabela 1, permitiu identificar que os estudantes concordaram que toda pessoa tem direito universal e integral à saúde, sendo dever do Estado efetivar esse direito. Em relação as ações judiciais relacionadas a saúde como forma de garantir o direito à saúde, os estudantes de enfermagem e direito se mostraram concordantes, sendo 4,37 e 4,61 as médias respectivamente.

Estes dados corroboram com o percentual obtido na questão três que analisa o conhecimento dos estudantes sobre as ações judiciais como garantia de um direito individual. No que se refere as ações judiciais para garantia de medicamentos de alto custo, os estudantes de enfermagem e direito se mostraram concordantes, contudo, os estudantes de medicina apresentaram média 3,59, demostrando-se imparciais em relação a afirmativa.

Quando questionados em relação ao Estado ser capaz de decidir e garantir leitos de internação, mesmo não vivenciando as realidades das instituições, o curso de direito apresentou a maior média do instrumento, assinalando que os estudantes se mostraram imparciais, enquanto o curso de enfermagem e medicina se mostraram discordantes. O nível de significância dessa questão foi de ,029, demonstrando que há diferença entre as médias nos três cursos. Já com relação a questão seis, ainda sobre leitos de internação, os estudantes de medicina concordam que as judicializações para garantia de leitos de internação podem gerar conflitos éticos. Quando comparado aos alunos dos outros cursos, o curso de medicina apresentou a maior média, por seguinte, o curso de enfermagem 3,77 e direito 3,6, sendo o nível de significância de ,000, confirmando as diferenças nas médias entre os três cursos.

Quando questionados acerca do conhecimento da população sobre as judicializações em saúde, os estudantes se mostraram discordantes, demostrando que esses acreditam que muitas pessoas ainda não são informadas sobre seus direitos, principalmente no que tange à saúde. Além disso, os estudantes concordam que os profissionais de saúde e do direito devem participar mais ativamente da divulgação de conhecimentos sobre judicialização, a fim de empoderar os indivíduos a efetivar seu direito à saúde. Essa questão apresenta nível de significância de ,046, assinalando a diferença entre as médias.

Em relação a formação dos Profissionais de Enfermagem e Medicina, os estudantes discordaram que a formação dos profissionais de Enfermagem e Medicina prepara os estudantes de forma satisfatória para compreensão das questões relacionadas à efetivação da garantia do direito à saúde. Já em relação a formação dos Profissionais de Direito, os estudantes se mostram discordantes em relação à questão do preparo dos profissionais do direito durante a formação para lidar com a judicialização na saúde. 
Tabela 1 - Análise da compreensão discente sobre a judicialização da saúde nos diferentes cursos.

\begin{tabular}{|c|c|c|c|c|c|}
\hline Fatores & Cursos & $\mathbf{N}$ & Média & DP & $\begin{array}{c}\text { Nível de } \\
\text { Significância }\end{array}$ \\
\hline \multirow{4}{*}{$\begin{array}{l}\text { Q1 Toda a pessoa tem direito universal e } \\
\text { integral à saúde, sendo dever do Estado } \\
\text { efetivar esse direito. }\end{array}$} & Enfermagem & 30 & 4,93 & .254 & \\
\hline & Medicina & 51 & 4,73 & 850 & \\
\hline & Direito & 36 & 4,97 &, 169 & \\
\hline & Total & 117 & 4,85 &, 594 & - \\
\hline \multirow{4}{*}{$\begin{array}{l}\text { Q2 As ações judiciais relacionadas à saúde } \\
\text { possibilitam a efetivação do direito à saúde. }\end{array}$} & Enfermagem & 30 & 4,37 & ,890 & \\
\hline & Medicina & 51 & 3,84 & 1,155 & \\
\hline & Direito & 36 & 4,61 & ,728 & \\
\hline & Total & 117 & 4,21 & 1,024 & - \\
\hline \multirow{4}{*}{$\begin{array}{l}\text { Q3 As ações judiciais efetivam o acesso à } \\
\text { saúde, porém de forma individual. }\end{array}$} & Enfermagem & 30 & 4,07 & 1,048 & \\
\hline & Medicina & 51 & 3,84 & 1,084 & \\
\hline & Direito & 36 & 4,11 & 1,008 & \\
\hline & Total & 117 & 3,98 & 1,050 & - \\
\hline Q4 A garantia de medicamentos de alto & Enfermagem & 30 & 4,30 &, 877 & \\
\hline custo por processos judiciais constitui-se & Medicina & 51 & 3,59 & 1,314 & \\
\hline em um direito do paciente e deve sempre & Direito & 36 & 4,58 & ,692 & \\
\hline ser fornecido, mesmo que gere impacto & Total & 117 & 4,08 & 1,131 & - \\
\hline \multicolumn{6}{|l|}{ financeiro para o Estado. } \\
\hline Q5 O Estado é capaz de decidir e garantir & Enfermagem & 30 & 2,90 & 1,373 & \\
\hline leitos de internação para requerentes & Medicina & 51 & 2,45 & 1,154 & \\
\hline mesmo não vivenciando as realidades das & Direito & 36 & 3,11 & 1,254 & \\
\hline instituições de saúde. & Total & 117 & 2,79 & 1,272 & 0,29 \\
\hline Q6 Internações por ordem judicial podem & Enfermagem & 30 & 3,77 & 1,194 & \\
\hline gerar conflitos éticos, pois nem sempre os & Medicina & 51 & 4,16 & 1,046 & \\
\hline requerentes são pacientes com risco de & Direito & 36 & 3,06 & 1,286 & \\
\hline vida. & Total & 117 & 3,72 & 1,245 &, 000 \\
\hline Q7 O acesso às ações e serviços de saúde & Enfermagem & 30 & 5,00 &, 000 & \\
\hline deve ser garantido a todas as pessoas, & Medicina & 51 & 4,94 & ,420 & \\
\hline independente de sexo, raça ou outras & Direito & 36 & 4,97 &, 167 & \\
\hline características sociais ou pessoais. & Total & 117 & 4,97 & ,292 & - \\
\hline Q8 Todas as pessoas possuem direito as & Enfermagem & 30 & 4,07 & 1,337 & \\
\hline ações e serviços de saúde, mas o Estado & Medicina & 51 & 4,51 & 809 & \\
\hline \multirow[t]{2}{*}{ deve investir onde a carência é maior. } & Direito & 36 & 4,22 & ,959 & \\
\hline & Total & 117 & 4,31 & 1,021 & - \\
\hline Q9 Toda sociedade está devidamente & Enfermagem & 30 & 1,57 & ,935 & \\
\hline informada sobre seus direitos em relação à & Medicina & 51 & 1,67 & 1,071 & \\
\hline saúde e sobre a possibilidade de & Direito & 36 & 1,56 & ,969 & \\
\hline judicialização. & Total & 117 & 1,61 & 1,000 & - \\
\hline Q10 Os profissionais de saúde e do direito & Enfermagem & 30 & 4,50 & 900 & \\
\hline devem participar na divulgação de & Medicina & 51 & 3,96 & 1,199 & \\
\hline conhecimentos sobre judicialização a fim & Direito & 36 & 4,47 & 1,158 & \\
\hline
\end{tabular}




$\begin{array}{lccccc}\text { de empoderar os indivíduos a efetivar seu } & \text { Total } & 117 & 4,26 & 1,138 & \text {,046 } \\ \text { direito à saúde. } & & & & & \\ \text { Q11 As demandas judiciais trazem } & \text { Enfermagem } & 30 & 3,87 & 1,137 \\ \text { consequências financeiras para as } & \text { Medicina } & 51 & 4,08 & , 913 \\ \text { instituições de saúde e para gestão } & \text { Direito } & 36 & 3,83 & 1,298 & - \\ \text { orçamentária do sistema de saúde. } & \text { Total } & 117 & 3,95 & 1,097 \\ \text { Q12 Os enfermeiros e médicos são } & \text { Enfermagem } & 30 & 2,47 & 1,224 \\ \text { preparados já na graduação para } & \text { Medicina } & 51 & 2,51 & 1,206 \\ \text { compreender as questões relacionadas a } & \text { Direito } & 36 & 2,72 & 1,210 \\ \text { efetivação da garantia do direito à saúde. } & \text { Total } & 117 & 2,56 & 1,206 & - \\ \text { Q13 Os profissionais do Direito são } & \text { Enfermagem } & 30 & 2,63 & 1,098 \\ \text { preparados já na graduação para lidar com a } & \text { Medicina } & 51 & 2,55 & 1,101 \\ \text { judicialização da saúde. } & \text { Direito } & 36 & 3,03 & 1,253\end{array}$

*nível de significância considerado: $\mathrm{p}<0,05$. Fonte: Autores.

A partir dos resultados apresentados neste estudo constatou-se que os estudantes concordam que toda pessoa tem direito à saúde, e que as ações judiciais possibilitam a garantia desse direito, exceto os estudantes de medicina que se mostraram imparciais no que se refere ao fato das ações judiciais possibilitarem a efetivação do direito à saúde. Esse entendimento acerca do direito à saúde é fundamental para garantia ao acesso integral, dado que o estudante reconhece o direito à saúde como um direito inalienável da pessoa. Desse modo, o cuidado para garantir o direito à saúde a todos os cidadãos necessita de esforços advindos de todos os profissionais, em especial aos profissionais de enfermagem, de modo que a assistência à saúde, a prevenção e a recuperação de agravos sejam todos baseados nos princípios norteadores do SUS, os quais são essenciais para a melhoria das condições de vida dos indivíduos (Cofen, 2016).

Os estudantes de medicina apresentam-se imparciais quando questionados sobre as ações judiciais relacionadas a saúde como forma de garantir o direito à saúde e como garantia de um direito individual. Esta percepção diferente pode estar relacionada ao fato dos mesmos já durante a graduação abordarem temas relacionados a judicialização, porém sempre de forma negativa, envolvendo implicações ao profissional principalmente no que tange erros médicos (Pinheiro, 2018). A maioria dos processos judiciais estão ligados a prescrições médicas e a urgência em obter determinado insumo, como medicamentos de alto custo, solicitações de tratamento médico hospitalar e negligência médica (Paixão, 2019).

Estudos tem evidenciado um aumento no número de processos jurídicos relacionados à saúde, o qual vem ocasionando grande impacto financeiro ao país. Em torno de 1 bilhão de reais são gastos para atender a 4.855 pacientes e

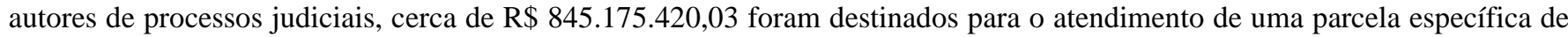
1.174 pacientes. Isto é, na judicialização, 24\% das demandas consumiram 77\% dos recursos públicos do Brasil (Brasil, 2017).

Conforme o relatório elaborado pelo Juiz Federal Clenio Jair Schulze, no ano de 2015, consta que mais de 854.000 processos judiciais ligados à saúde, sendo desses, 200.090 ligados ao fornecimento de medicamentos, 61.655 a serviços de saúde, 60.696 ao tratamento médico-hospitalar e 38.810 relacionados ao erro médico (Schulze, 2016). Portanto, por mais que o sistema de saúde forneça recursos de forma universal, esses, contudo, não conseguem atender as demandas de todos os usuários, gerando casos de judicialização (Leite \& Bastos, 2018).

Com relação as ações judiciais para garantia de medicamentos de alto custo, os estudantes de enfermagem e direito se mostraram concordantes, contudo, novamente os estudantes de medicina apresentaram-se imparciais. Nesta perspectiva, o 
aumento dos custos de novos medicamentos atua sobre a assistência farmacêutica do SUS, ao passo que as ações judiciais obrigam o fornecimento dessas medicações pelos órgãos públicos, baseados na premissa constitucional do acesso universal à saúde. Através dessas ações, o judiciário favorece a inserção de medicamentos não padronizados no SUS, assim como daqueles que não estão presentes nos protocolos elaborados pelo Ministério da Saúde (Paula \& Bittar, 2017). Em vista disso, pode se observar que as decisões judiciais podem ocasionar desorganização na gestão dos serviços de saúde, pois acabam interferindo no planejamento das ações (Ramos et al., 2016).

Da mesma forma que os estudantes se mostraram imparciais em relação as judicializações para garantia de leitos de internação, os estudiosos acreditam que o uso da via judicial encontra-se como um contrassenso, visto que as decisões judiciais garantem o acesso aos serviços de saúde, mas também fazem com que não haja o cumprimento das premissas do SUS, dado que o caráter de atendimento é predominantemente individual (Ramos et al., 2016).

Ao se considerar o direito à saúde como um direito social, no qual objetiva a redução das desigualdades, o acesso a leitos de internação por via judicial acaba indo contra o princípio da igualdade, previsto na Constituição Federal de 1988, pois o acesso aos leitos de internação é garantido de forma individual (Ramos et al., 2016). Desta forma, as judicializações relacionadas ao acesso mostram-se duvidosas, ao ponto que garantem a efetivação do direito à saúde, porém ao mesmo tempo comprometem as políticas públicas de saúde no Brasil.

Ao se utilizar a via judicial como principal meio para garantir o acesso aos serviços de saúde, o sistema judiciário passa a se tornar mais uma porta de entrada ao sistema de saúde, comprometendo assim com as bases teóricas e conceituais que norteiam o acesso ao sistema de saúde do Brasil (Ramos et al., 2016). A judicialização em saúde pode favorecer a criação de um SUS de duas portas: uma destinada para a população que recorre ao Judiciário e obtém acesso a recursos do estado para atendimento de seus problemas de saúde; e outra voltada para o restante dos cidadãos, que acabam ficando com o acesso mais limitado ainda, em virtude desse redirecionamento de recursos para o beneficiamento dos indivíduos que acabaram acessando a porta alternativa (Ferraz \& Wang, 2014).

Assim, através da judicialização os princípios norteadores do SUS podem ficar descaracterizados. O princípio da integralidade é interferido quando as ações judiciais não levam em consideração o contexto social do solicitante, sendo que algumas vezes o problema não corresponde somente à esfera da saúde e sim a outras áreas. Além disso, o princípio da universalidade também é afetado, visto que as demandas judiciais garantem o acesso à saúde, porém essas ações visam o atendimento majoritariamente individual, descumprindo assim as premissas estipuladas pelo SUS. Ademais, a equidade na judicialização também se dissocia dos preceitos elencados pelo SUS, uma vez que as decisões judiciais perpetuam ainda mais as desigualdades em saúde, dado que é apenas o grupo de indivíduos que recorreram ao Judiciário tem garantia ao acesso ao recurso solicitado (Ramos et al., 2017).

Nesse sentido, a intervenção judicial no campo de saúde acarreta tensões no dia a dia dos profissionais de saúde, visto que a influência de instâncias e sujeitos que não fazem parte dos serviços de saúde impactam negativamente na rotina de trabalho (Ramos et al., 2017). Além disso, a literatura traz que há um desconforto por parte dos profissionais de saúde em aceitar as decisões judiciais, dado que quando os leitos são requisitados de forma judicial, nem sempre os critérios para a obtenção do leito são respeitados, logo, há dificuldade em estabelecer um diálogo entre a equipe de saúde e o Judiciário (Leite \& Bastos, 2018; Bohomol, Freitas \& Cunha, 2016).

Por isso, destaca-se a importância de durante a graduação provocar reflexões nos estudantes dos cursos da saúde e do direito sobre a judicialização da saúde, pois impõem-se uma forma de reafirmar os valores fundamentais de uma profissão e prática social que busca efetivar, em todos os planos, o bem-estar dos indivíduos (Carvalho \& David, 2013). Além disso, é uma maneira de preparar esses estudantes para lidar com questões que tangem a saúde, de modo que esses venham participar da divulgação de conhecimentos sobre judicialização, com o intuito de empoderar os indivíduos acerca do direito à saúde. 
Com relação as respostas na formação dos profissionais, os estudantes discordaram que a formação acadêmica e profissional auxilia na compreensão das questões relacionadas à judicialização da saúde. Neste sentido, essas respostas negativas relacionadas ao preparo profissional podem sinalizar que há uma falta, nos profissionais da saúde, de conhecimentos das leis implicadas na regulamentação de suas profissões, além da necessidade de os profissionais do Direito desenvolverem uma visão multidisciplinar ao conduzirem corretamente um processo judicial, cercando-se de pessoas habilitadas e capacitadas para realizarem julgamentos técnicos.

Desse modo, a falta de abordagem desse conteúdo no decorrer da formação pode gerar futuros profissionais com competências pouco desenvolvidas para decidir questões de judicialização relacionadas ao âmbito da saúde (Andia \& Lamprea, 2019). Ademais, esse sentimento de despreparo para lidar com as judicializações pode demostrar fragilidades na formação dos graduandos, na qual deve ser voltada inicialmente para as bases holísticas de cada curso.

\section{Conclusão}

Ao analisar o conhecimento discente sobre a formação acadêmica para a judicialização da saúde de alunos de cursos de graduação em enfermagem, medicina e direito é uma forma de investigar se esses estudantes estão preparados para lidar com questões jurídicas e processuais.

Destacam-se os resultados em relação a formação dos profissionais da enfermagem e medicina e formação dos profissionais do direito, nos quais os estudantes discordam que a formação dos profissionais do direito, enfermagem e medicina auxilia na compreensão das questões relacionadas à judicialização da saúde. Por isso, salienta-se a importância da abordagem dessas questões durante a formação acadêmica de enfermeiros, médicos e advogados, com o intuito de desenvolver o pensamento ético, humano, crítico e reflexivo, capaz de transformar a realidade de indivíduos e coletividades por meio da conscientização sobre o acesso à informação e ao direito à saúde.

Ressalta-se como limitação do estudo, a escassez de pesquisas que abordem a judicialização da saúde nos três cursos estudados. Assim, espera-se que com os resultados apresentados nesse trabalho possa contribuir de forma significativa, possibilitando que outras pesquisas sejam realizadas em diferentes universidades e que questões como a judicialização comecem a ser discutidas nos cursos de graduação. Por fim, reafirma-se a importância da realização de mais estudos que investiguem a temática.

\section{Referências}

Andia, T. S. \& Lamprea, E. (2019). A judicialização da saúde é prejudicial à equidade? Uma revisão de escopo. Int J Equity Health; 18 (1), 61.10 .1186 / s12939-019-0961-y.

Appolinário, F. (2012). Metodologia da ciência: filosofia e prática da pesquisa. (2a ed.), Cengage Learning.

Bohomol, E., Freitas, M. A. O., \& Cunha, I. C. K. O. (2016). Ensino da segurança do paciente na graduação em saúde: reflexões sobre saberes e fazeres. Revista Interface (Botucatu);20 (58),727-41. https://doi.org/10.1590/1807-57622015.0699.

Calliari, G. \& Teixeira, A. V. (2017). A judicialização da saúde e a má distribuição de recursos para medicamentos de baixa escala e/ou alto custo. Revista Eletrônica Direito e política; 12 (1). https://doi.org/10.14210/rdp.v12n1.p202\%20-\%20220.

Carvalho, E. C., \& David, H. M. S. L. (2013). Judicialização da saúde, problema e solução: questões para a enfermagem. Rev. enferm. UERJ;21(4):546-50. https://www.e-publicacoes.uerj.br/index.php/enfermagemuerj/article/view/10015/7809

Conselho federal de enfermagem. Enfermagem em defesa da saúde como direito constitucional. (2016). COFEN. http://www.cofen.gov.br/enfermagememdefesa-da-saude-como-direito-constitucional_43418.html

Ferraz, O. L. M., \& Wang, D. W. L. (2014). As Duas Portas do SUS. Folha de São Paulo, São Paulo, Opinião. https://www1.folha.uol.com.br/paywa 11/login.shtml?https://www1.folha.uol.com.br/opiniao/2014/06/1472761-octavio-ferraz-e-daniel-wang-as-duas-portas-dosus.shtml

Gray, D. E. (2012). Pesquisa no Mundo Real. (2a ed.), Penso. 
Research, Society and Development, v. 10, n. 2, e51410212795, 2021

(CC BY 4.0) | ISSN 2525-3409 | DOI: http://dx.doi.org/10.33448/rsd-v10i2.12795

Hill, M. M., \& Hill, A. (2012). Investigação por questionário. Editora Sílabo.

Leite, I. C., \& Bastos, P. R. H. O. (2018). Judicialização da saúde: aspectos legais e impactos orçamentários. Revista Argumentum; 10(1):102-117. https://dialnet.unirioja.es/servlet/articulo?codigo $=6545987$

Lopes, L., Acurcio, F. A., Diniz, S. D., Coelho, T. L., \& Andrade, E. (2019). Distribuição (des) eqüitativa dos recursos em saúde e a judicialização da saúde: 10 anos de experiência no Brasil. Jornal internacional para a equidade em saúde;18(1), 10.

Ministério da Saúde (BR). (2017). Consultoria Jurídica da Advocacia-Geral da União junto ao Ministério da Saúde. Manual de Judicialização da saúde no Brasil: Desafios para a mediação. Ministério da Saúde. http://portalarquivos2.saude.gov.br/images/pdf/2017/maio/17/JUDIC IALIZACAO\%20DA\%20SAUDE\%20NO\%20BRASIL\%20Desafios\%20para\%20a\%20mediacao.pdf

Oates, K. et al. (2018). Changing medical student attitudes to patient safety: a multicentre study. BMC Medical Education; 18 (205) https://doi.org/10.1186/s12909-018-1313-0.

Paixão, A. L. S. (2019). Reflections on the judicialization of the right to health and its implications in the SUS. Ciência \& Saúde Coletiva;24(6),2167-2172 https://doi.org/10.1590/1413-81232018246.08212019.

Paula, C. E. A., \& Bittar, C. M. L. (2017). Judicialização da saúde e seus reflexos na gestão do sistema único de saúde (SUS). Revista de Direitos Humanos e Efetividade;3(1), 19-41.

Pinheiro, R. A. A. (2018). judicialização da medicina. Jus.com.br. https://jus.com.br/artigos/57500/a-judicializacao-da-medicina

Ramos, R. S., Gomes, A. M. T., Guimarães, R. M., \& Santos, E. I. (2017). A judicialização da saúde contextualizada na dimensão prática das representações sociais dos profissionais de saúde. Revista De Direito Sanitário;18(2), 18-38. http://dx.doi.org/10.11606/issn.2316-9044.v18i2p18-38.

Ramos, R. S., Gomes, A. M. T., Oliveira, D. C., Marques, S. C., Spindola, T., \& Nogueira, V. P. F. (2016). Access the Unified Health System actions and services from the perspective of judicialization.Revista Latino-Americana de Enfermagem, 24, e2797. https://doi.org/10.1590/1518-8345.1012.2689.

Schulze, C. J. (2016). Novos números sobre a judicialização da saúde. http://emporiododireito.com.br/leitura/novos-numeros-sobre-a-judicializacao-da-saudepor-clenio-jair-schulze

Souza, V. S. et al. (2018). Erros de enfermagem no processo de medicação: análise de mídia eletrônica televisiva. Revista Esc Anna Nery;22(2). https://doi.org/10.1590/2177-9465-ean-2017-0306

Travassos, D. V., Ferreira, R. C., Vargas, A. M. D., Moura, R. N. V., Conceição, E. M. A. C, \& Marques, D. F. et al. (2013). Judicialização da Saúde: um estudo de caso de três tribunais brasileiros. Ciência \& Saúde Coletiva;18(11):3419-3429. https://doi.org/10.1590/S1413-81232013001100031

Wegner, W., Silva, S. C., Kantorski, J. C., Predebon, C. M, Sanches, M. O., \& Pedro, E. N. R. (2016). Educação para cultura da segurança do paciente: Implicações para a formação profissional.Esc Anna Nery; 20(3):e20160068. http://dx.doi.org/10.5935/1414-8145.20160068 